\title{
Author Correction: End-to-end lung cancer screening with three-dimensional deep learning on low-dose chest computed tomography
}

Diego Ardila D, Atilla P. Kiraly, Sujeeth Bharadwaj, Bokyung Choi, Joshua J. Reicher, Lily Peng, Daniel Tse (D), Mozziyar Etemadi (D), Wenxing Ye, Greg Corrado, David P. Naidich and Shravya Shetty

Correction to: Nature Medicine https://doi.org/10.1038/s41591-019-0447-x, published online 20 May 2019.

In the version of this article originally published, there was an error in the phrase "This dataset contained 1,739 cases (27 cancer-positives)" in the main text. The number 1,739 should have been 1,139. There was also an error in the Fig. 4c legend. In the phrase "comprising $n=1,739$ cases", the number 1,739 again should have been 1,139. Additionally, in the Extended Data Fig. 5 legend, the phrase "AUC curve for the independent data test set with $n=1,739$ cases" contained the same error. The number should have been 1,139 instead of 1,739. The errors have been corrected in the HTML and PDF versions of this article.

\section{Author Correction: Farm-like indoor microbiota in non-farm homes protects children from asthma development}

Pirkka V. Kirjavainen (D), Anne M. Karvonen (D), Rachel I. Adams, Martin Täubel(D), Marjut Roponen, Pauli Tuoresmäki, Georg Loss, Balamuralikrishna Jayaprakash, Martin Depner, Markus Johannes Ege (D), Harald Renz, Petra Ina Pfefferle, Bianca Schaub, Roger Lauener (D), Anne Hyvärinen, Rob Knight (DD, Dick J. J. Heederik, Erika von Mutius and Juha Pekkanen

Correction to: Nature Medicine https://doi.org/10.1038/s41591-019-0469-4, published online 17 June 2019.

In the version of this article originally published, several competing interests for Erika von Mutius were missing. This sentence was added to the competing interests section: "E.v.M. is an inventor on the patents EP1637147, EP1964570, LU101064 and EP1411977; E.v.M. is an inventor on and has received royalties from the patent EP2361632." The error has been corrected in the HTML and PDF versions of this article.

\section{Publisher Correction: Combined BRAF and MEK inhibition with PD-1 blockade immunotherapy in BRAF-mutant melanoma}

Antoni Ribas (D), Donald Lawrence, Victoria Atkinson, Sachin Agarwal, Wilson H. Miller Jr, Matteo S. Carlino, Rosalie Fisher, Georgina V. Long DD, F. Stephen Hodi, Jennifer Tsoi, Catherine S. Grasso, Bijoyesh Mookerjee, Qing Zhao, Razi Ghori, Blanca Homet Moreno, Nageatte Ibrahim and Omid Hamid

Correction to: Nature Medicine https://doi.org/10.1038/s41591-019-0476-5, published online 06 June 2019.

In the version of this article originally published, there was an error in the Fig. 1c key. The label for the light blue color was "Time since last scan". It should have been "Time to last scan". The error has been corrected in the HTML and PDF versions of this article. 